\title{
Biocompatible Lipopeptide-Based Antibacterial
}

\section{Hydrogel}

Anindyasundar Adak, ${ }^{1}$ Subhajit Ghosh, ${ }^{1}$ Varsha Gupta, ${ }^{1}$ Surajit Ghosh ${ }^{1,2}$

1. Organic \& Medicinal Chemistry Division, CSIR-Indian Institute of Chemical Biology, 4 Raja S. C. Mullick Road, Jadavpur, Kolkata-700032, West Bengal, India. Fax: +91-33-24735197/0284; Tel: +91-33-2499-5872

2. Academy of Scientific and Innovative Research (AcSIR), Ghaziabad - 201002, India 


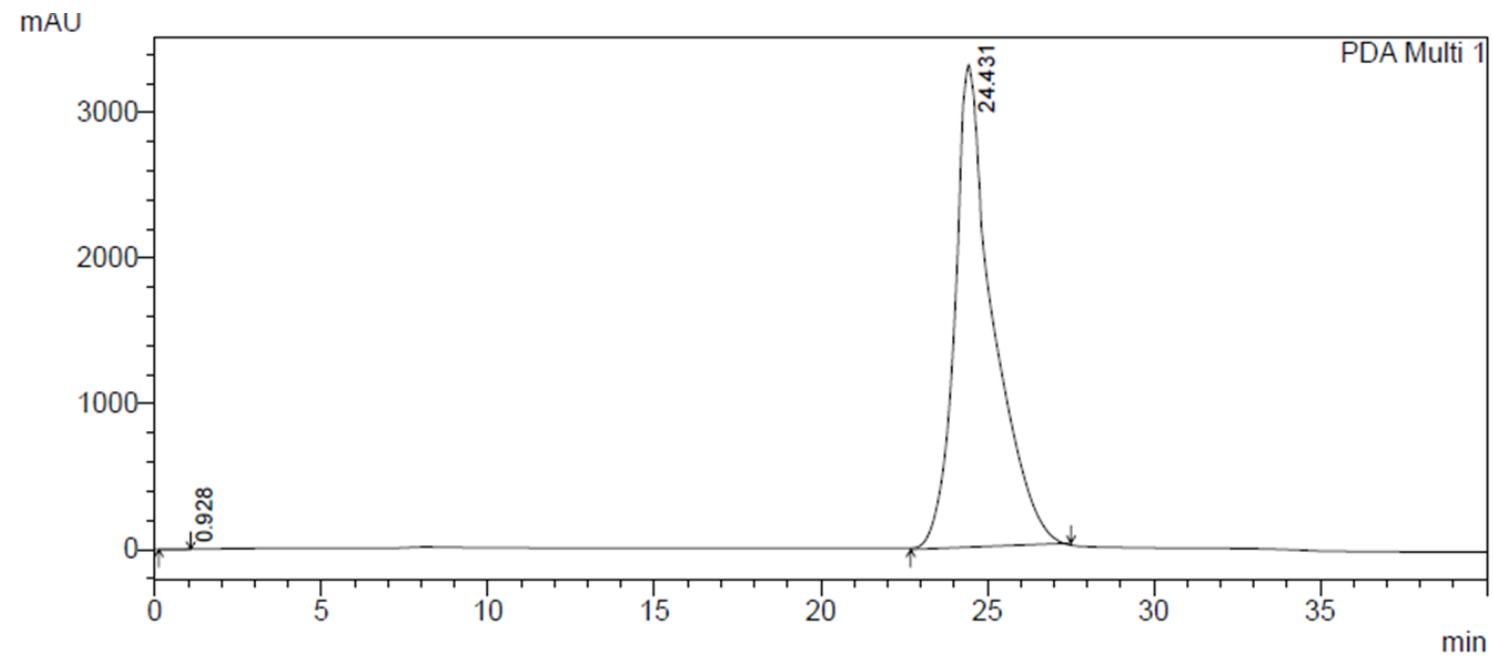

1 PDA Multi 1/210nm 4nm

PeakTable

PDA Ch1 210nm 4nm

\begin{tabular}{|r|r|r|r|r|r|}
\hline \multicolumn{1}{|c|}{ Peak\# } & Ret. Time & \multicolumn{1}{c|}{ Area } & \multicolumn{1}{c|}{ Height } & \multicolumn{1}{c|}{ Area \% } & \multicolumn{1}{c|}{ Height \% } \\
\hline 1 & 0.928 & 19260 & 356 & 0.007 & 0.011 \\
\hline 2 & 24.431 & 265296315 & 3316881 & 99.993 & 99.989 \\
\hline Total & & 265315575 & 3317237 & 100.000 & 100.000 \\
\hline
\end{tabular}

Figure S1: HPLC chromatography of the PA-NV molecule. 


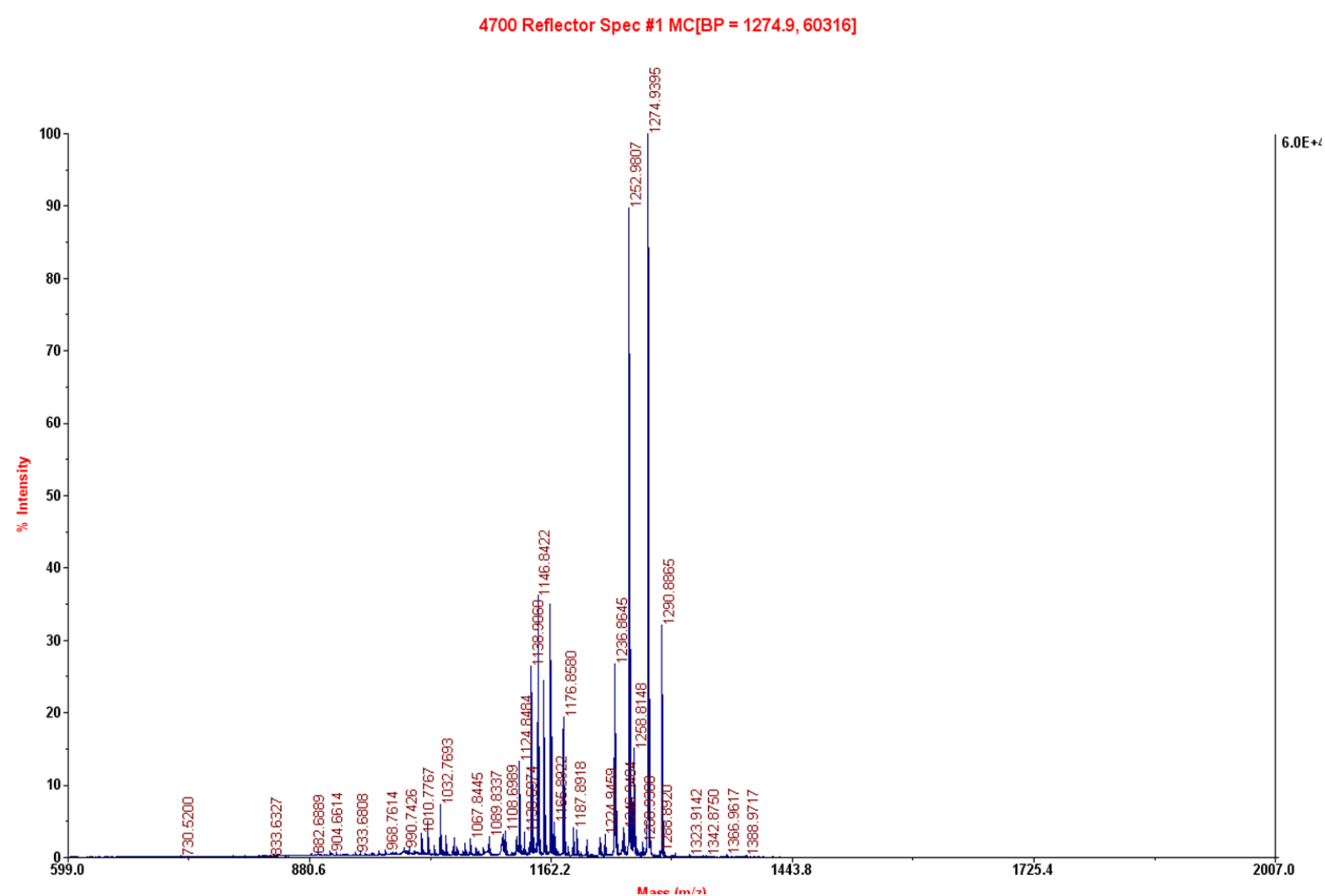

Figure S2: MALDI-TOF Mass spectrometry of PA-NV molecule $(\mathrm{M}=1252, \mathrm{M}+\mathrm{Na}=$ $1274, \mathrm{M}+\mathrm{K}=1290$ ). 


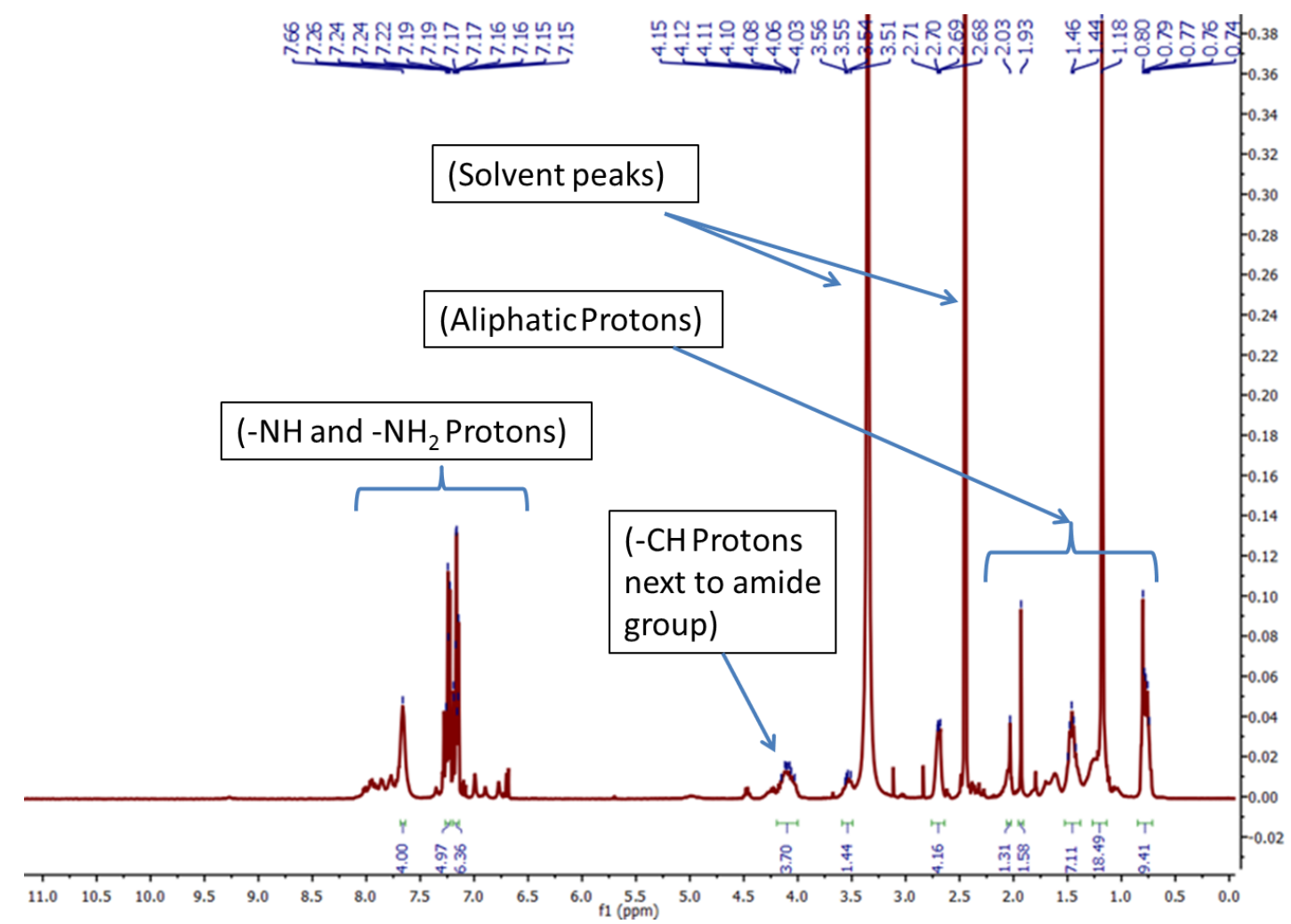

Figure S3: ${ }^{1} \mathrm{H}$ NMR spectroscopy of PA-NV molecule in $\mathrm{d}^{6}$ DMSO. 


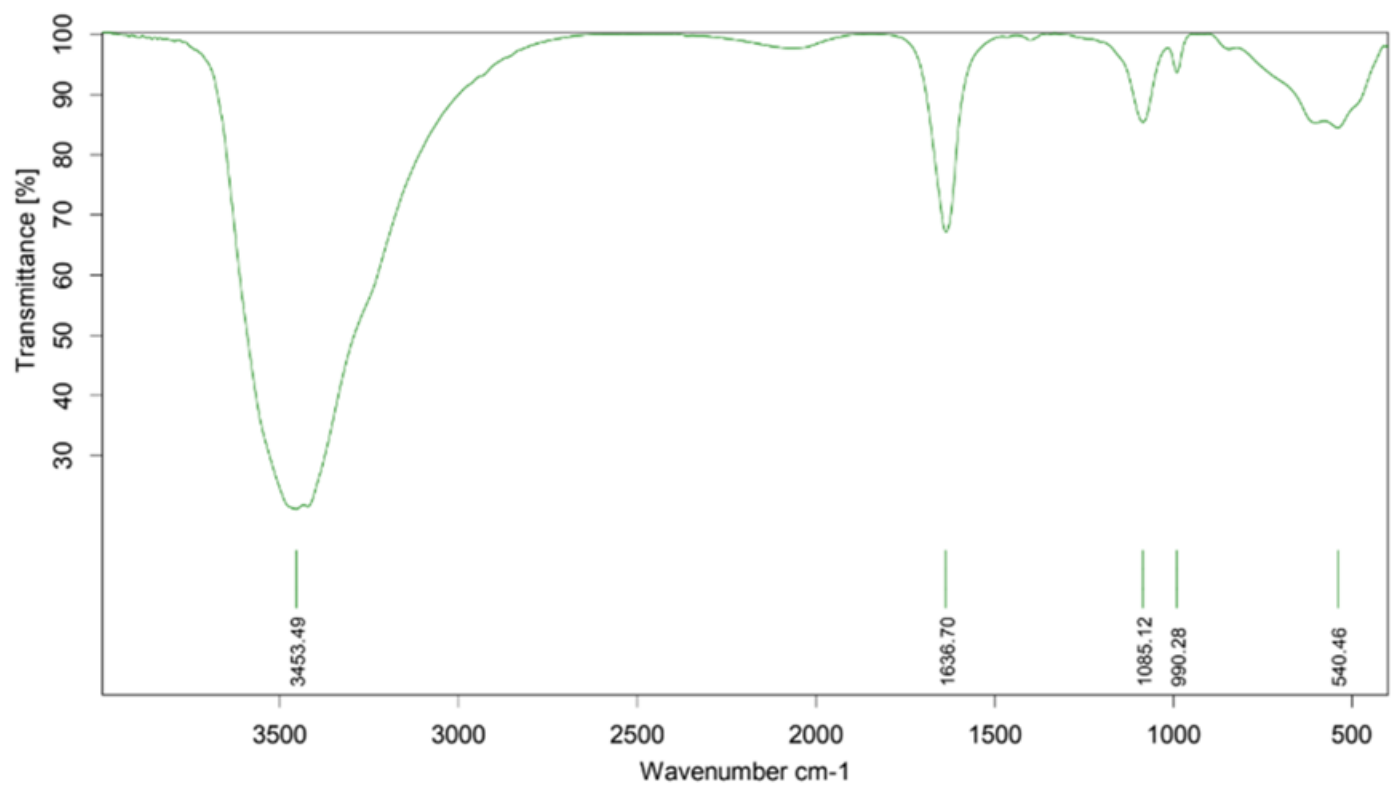

Figure S4: FT-IR spectra of 2 wt\% PA-NV hydrogel showing amide-I peak at $1635 \mathrm{~cm}^{-1}$, indicating formation of $\beta$-sheet secondary structure. 


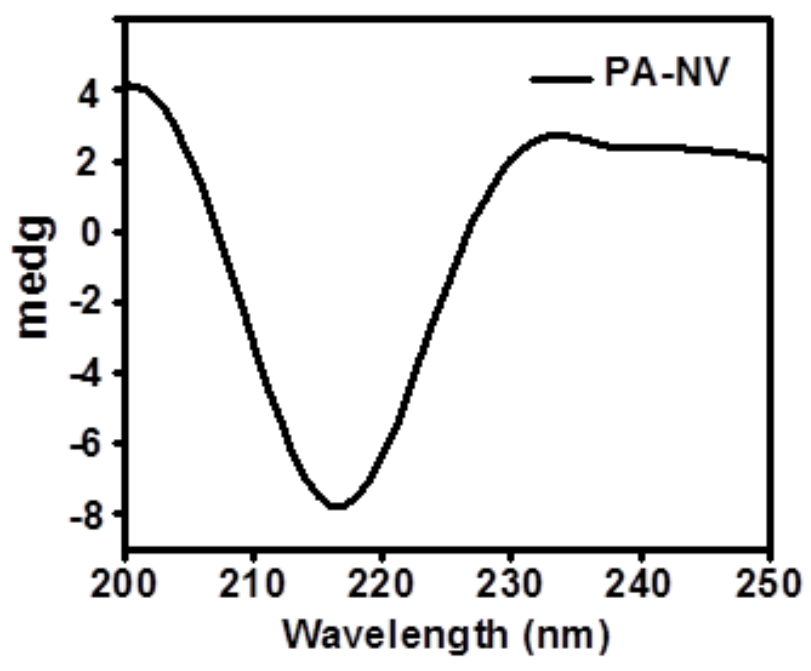

Figure S5: Circular dichroism (CD) experiment: Graph showing signature peak of $\beta$-sheet secondary structure at $216 \mathrm{~nm}$ of $2 \mathrm{wt} \%$ PA-NV hydrogel. 

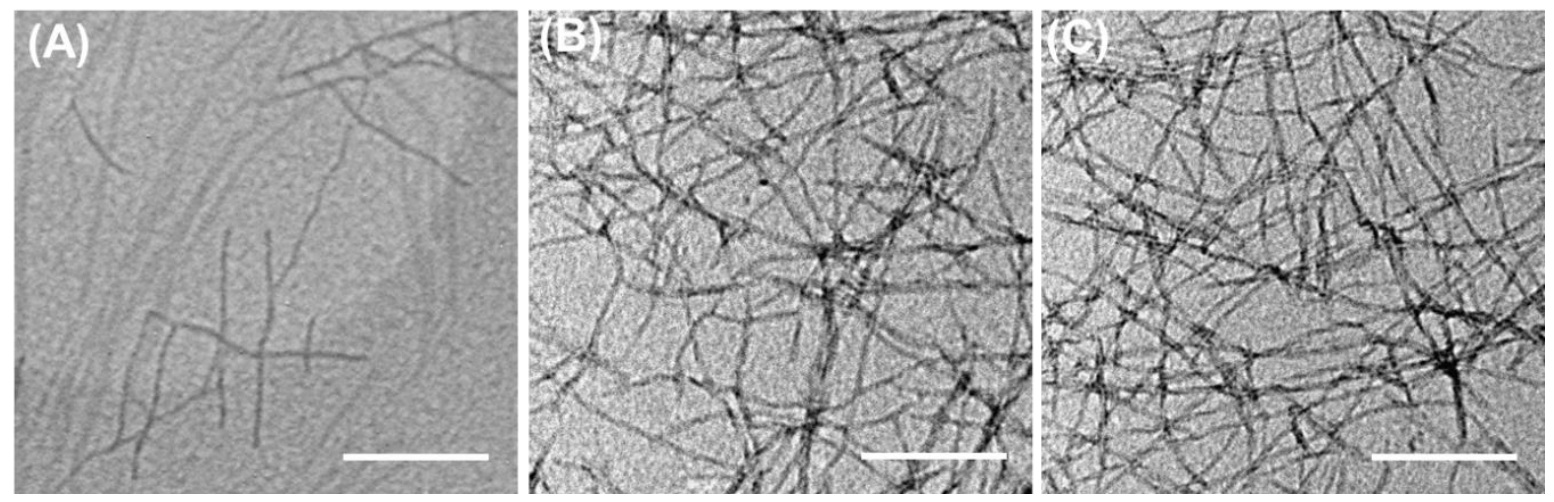

Figure S6: Transmission Electron Microscopy (TEM) study. Image represents weak, fragile nano-fiber of PA-NV before gelation (A), nano-fiber of $1 \mathrm{wt} \%$ hydrogel (B), more dense and intense nano-fiber of $2 \mathrm{wt} \%$ hydrogel (C). Scale bar corresponds to $400 \mu \mathrm{m}$. 


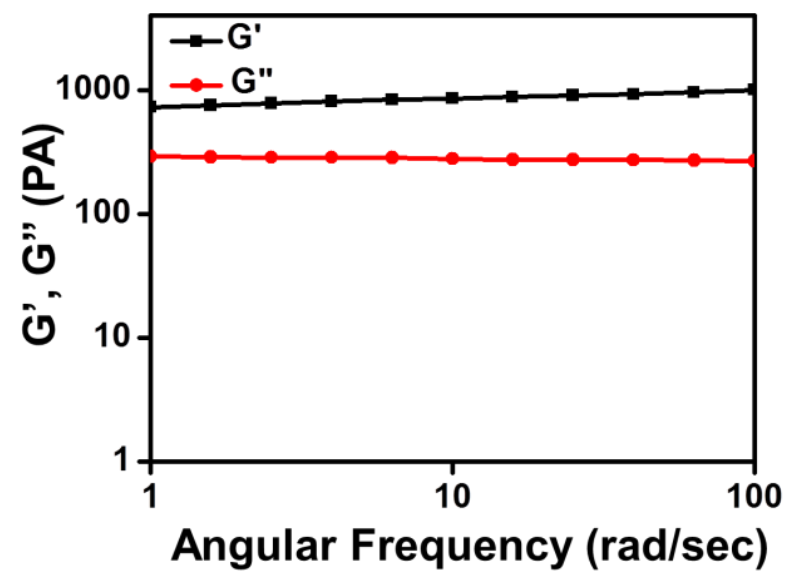

Figure S7: Frequency sweep rheology study of 4 wt\% PA-NV hydrogel over 100 seconds ( $G^{\prime}$ and $G^{\prime \prime}$ represents storage and loss modulus). The experiment performed at room temperature $25^{\circ} \mathrm{C}$. 


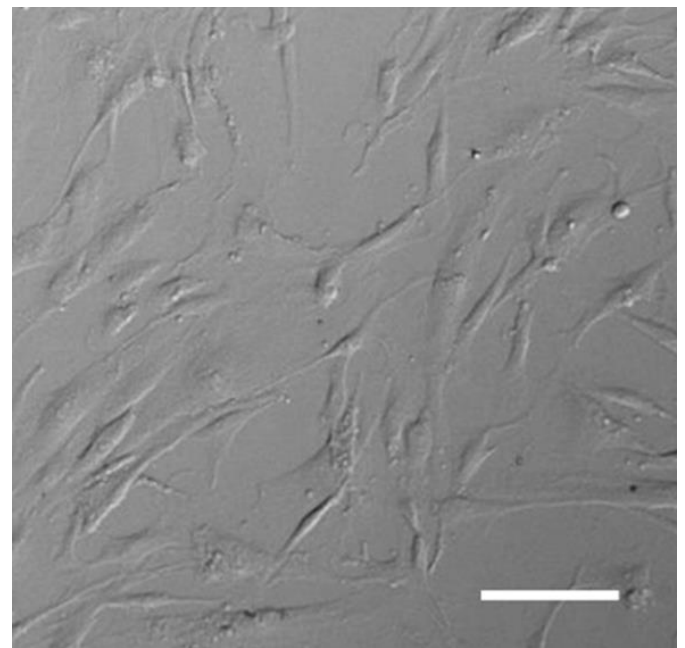

Figure S8: Control cell morphology of HeLa cell cultured without PA-NV hydrogel after 24 hr. incubation. 


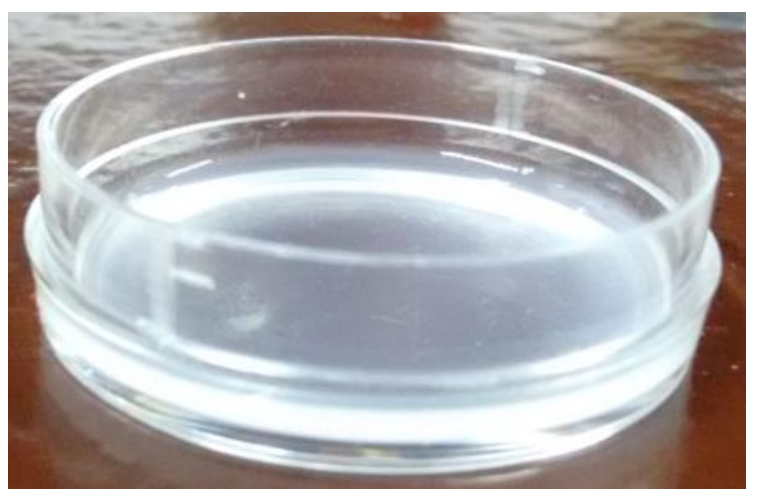

Figure S9: Surface coating of the round disk by PA-NV hydrogel. 


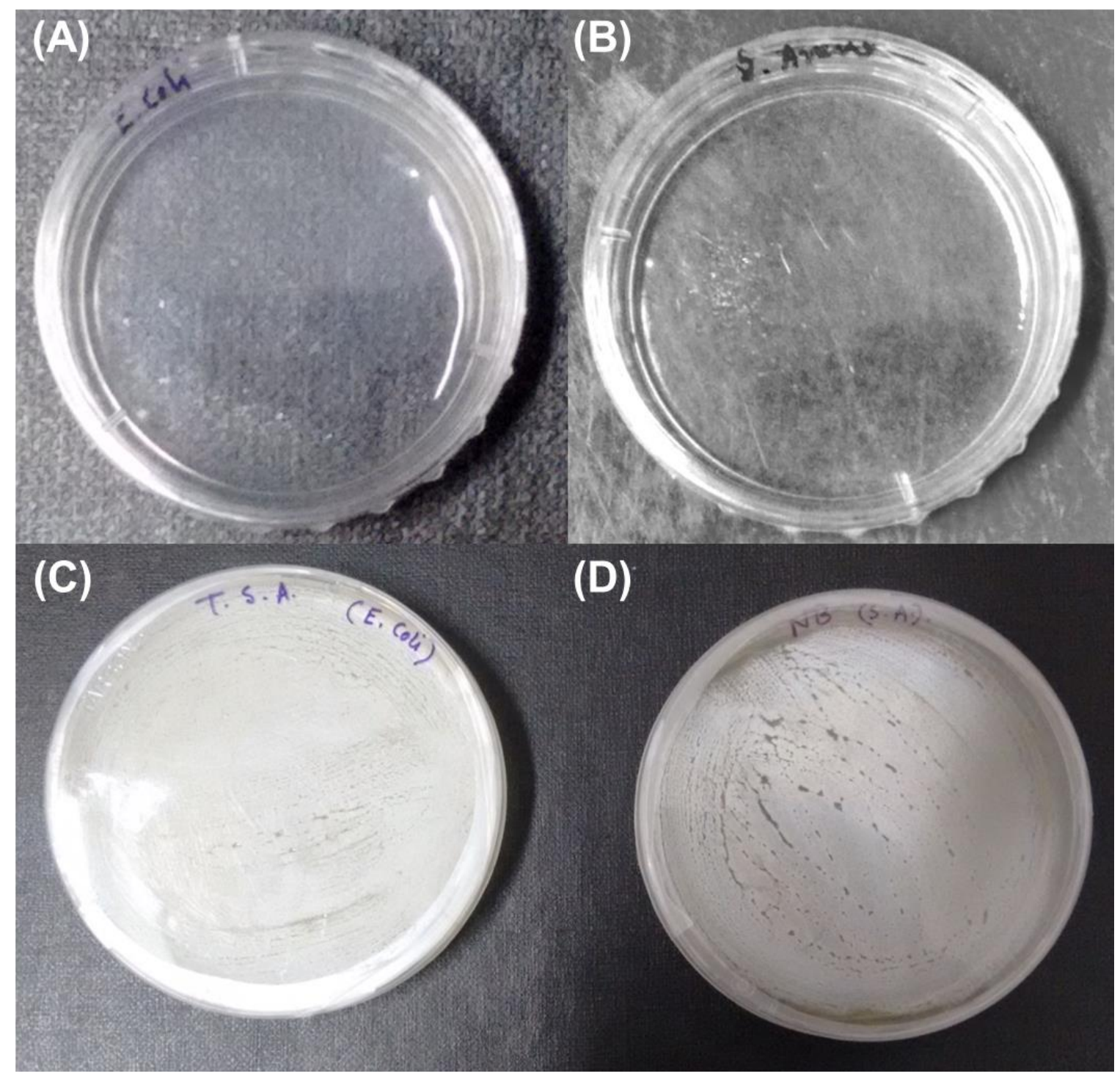

Figure S10: Colony inhibition study of $E$. coli (A) S. aureus (B) by hydrogel surface (C, D) Control. 

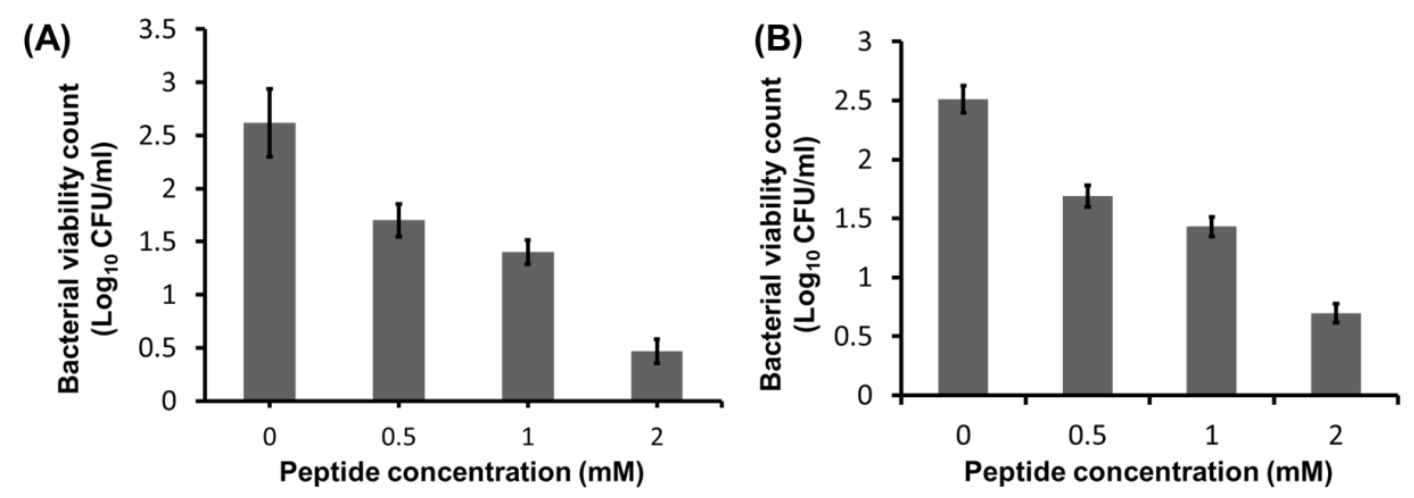

Figure S11: Colony count assay of E. coli (A) S. aureus (B) by PA-NV. 